\title{
Hypothermia as anesthesia
}

Like many other academic institutions, Great Eastern University used the pain and distress categories identified in the USDA Annual Report to classify the level of pain or distress experienced by all animals in the school's biomedical research program, not just those species covered by the Animal Welfare Act and its regulations. Anesthesia induced by hypothermia was considered to be USDA category D (pain or distress alleviated by drugs). For years, the university had considered hypothermia an acceptable means of anesthesia for neonatal rats and mice less than one week of age. This technique was used to anesthetize pups prior to decapitation and for certain surgical procedures when inhalant or parenteral anesthetics were contraindicated by the research.
Over time, as the composition of the IACUC changed, so did attitudes toward using hypothermia. The issue was brought to a head when Jonathan Bellamy, a neuroscientist on the committee, questioned whether hypothermia should be considered a drug. He believed it was necessary to consider it a drug in order for animals subjected to hypothermia-induced anesthesia to be placed in category $\mathrm{D}$; otherwise, he said, using the USDA's definition, animals anesthetized by hypothermia should be in category E (pain or distress unalleviated by drugs). The IACUC chairman, Larry Covelli, said that the argument was immaterial because use of the USDA categories was a convenience for the IACUC rather than a legal requirement. But
Bellamy persisted. First, he said that the issue should be resolved because it could easily involve newborn hamsters or other species covered by the USDA. Second, he questioned whether hypothermia itself had a painful induction and recovery process, even if the committee assumed that there was satisfactory anesthesia at the endpoint. "Remember," he said, "in humans, hypothermia is almost always used as an adjunct to general anesthesia, not as the sole means of anesthesia." Finally, he said, he was not confident whether the hypothermic animal was paralyzed or anesthetized.

What do you and your IACUC think? Is hypothermia an acceptable means of anesthesia if other means cannot be used? If used, should it be considered category D or E?

\section{RESPONSE}

\section{The rodent species is immaterial}

\section{Joan Richerson, MS, DVM, MS, DACLAM \& Barbara Beatty, BS}

Great Eastern University chose to use the USDA Annual Report's pain and distress categories for classifying the level of pain and distress experienced by all animals used in research; therefore, it is immaterial whether the species is USDA-regulated. Bellamy questioned whether hypothermia could be considered a drug. We contend that it is. A drug is defined by Dorland's Medical Dictionary as any medicinal substance ${ }^{1}$. If an ice water bath is used to induce hypothermia anesthesia, then it qualifies as a drug using Dorland's definition. Hypothermia has long been an accepted method of anesthesia for neonatal rodents when other methods are contraindicated. As an anesthetic, hypothermia works by depressing neural conduction and synaptic transmission in the central nervous system and by reducing conduction velocity in peripheral nerves ${ }^{2}$. Local anesthetics such as lidocaine hydrochloride depress or block nerve conduction when applied at appropriate concentrations ${ }^{3}$. Hypothermia by rapid chilling essentially has the same effect. Therefore, anesthesia induced by hypothermia would be appropriately placed in USDA category D (pain or distress alleviated by drugs). The fundamental question is under what circumstances (if any) the use of hypothermia anesthesia is reasonable and defensible.

As Bellamy pointed out, hypothermia is painful in humans and animals, especially adults. The age and size of the animal as well as the method of induction are important determinants in the animal's response to cold. Rodents less than a week of age cannot effectively regulate their body temperature; hence, their body temperature varies with the ambient temperature. In contrast to adult or larger animals, the small size of neonatal rodents allows for rapid skin and core body cooling. The method of hypothermia induction also affects the degree of discomfort experienced. Insulating rat pups in a latex sleeve rather than exposing them directly to an ice bath has been reported to lessen vocalization and struggling 4 . Presumably, as in humans, slowing the rate of chilling will reduce cold-induced pain ${ }^{5}$.

The concerns Bellamy raised about hypothermia are thought-provoking and should prompt the Greater Eastern University IACUC to re-evaluate its position on hypothermia anesthesia. The IACUC should consider approving hypothermia as a method of anesthesia only when the principal investigator can scientifically document that other methods are unacceptable. Second, the IACUC should develop a standard operating procedure for appropriate hypothermia induction. The IACUC may also want to observe the procedure of hypothermia anesthesia periodically to ensure that it is being done properly. These measures will help ensure that the use of hypothermia anesthesia at Great Eastern University is humane, appropriate and defensible.

1. Dorland's Pocket Medical Dictionary 22nd edn. (WB Saunders, Philadelphia, 1977).

2. Phifer, C.B. \& Terry, L.M. Use of hypothermia for general anesthesia in preweanling rodents. 